\title{
Eating alone, parents' marital status, and use of radio and girls' magazines were risk factors for eating disorders
}

Sources of funding: Fundación Echebano; Spanish Department of Health;Banco

Santander-Central-

Hispano.

For correspondence:

Dr A $M$

Martínez-González,

University of Navarra

Pamplona, Spain

mamartinez@unav.es

Martínez-González MA, Gual P,Lahortiga F, et al. Parental factors, mass media influences, and the onset of eating disorders in a prospective population-based cohort. Pediatrics 2003;111:315-20.

QUESTION: Are parental, mass media, sociodemographic, and psychosocial variables associated with an increased risk of developing an eating disorder (ED) in girls?

Design

Community cohort study with 18 months of follow up.
Setting

Navarra, Spain.

\section{Risk factors for developing an eating disorder}

\section{Risk factors} Adjusted odds ratio $(95 \% \mathrm{Cl}) \dagger$

\begin{tabular}{ll} 
Parents' marital status (other $v$ married) & $1.97(1.10$ to 3.51$)$ \\
\hline Eating alone (yes $v$ no) & $2.94(1.88$ to 4.60$)$ \\
\hline Reading girls' magazines $(<$ weekly $v \geq$ weekly) & $1.42(0.91$ to 2.2$) \ddagger$ \\
\hline Listening to radio $(\leq 1 \mathrm{~h} / \mathrm{d} v>1 \mathrm{~h} / \mathrm{d})$ & $1.55(1.01$ to 2.40$)$ \\
\hline
\end{tabular}

tOdds ratios were adjusted for all variables in table as well as age, body mass index, self esteem, and socioeconomic status.

‡Not significant.

\section{COMMENTARY}

Martínez-González et al add to the prospective research that explores how risk factors promote and/or maintain EDs. This study is consistent with other evidence regarding the influence of print media exposure and the associated risk of ED development. ${ }^{1}$ However, research examining television exposure is less consistent, ${ }^{2-3}$ and this inconsistency highlights the need for further examination of other factors that may interact with this medium (eg, peer groups and type of television programme). Martínez-González et al also found associations between variables such as parental marital status and solitary eating and ED onset that have not been widely shown, ${ }^{4}$ and further exploration of these associations is needed.

The study's findings are strengthened by its design. Initial levels of eating pathology were controlled for at baseline assessment, ensuring that increases in EDs were not a consequence of initial, marked disturbances. The control of confounders (eg, age at baseline assessment and socioeconomic status) adds confidence to the correlations. The prospective nature of this study and its use of logistic modelling ensure that potential interaction among factors could be investigated. However, as with all correlational research, findings do not necessarily connote causation and are vulnerable to other explanations. Solitary eating, for example, may reflect symptoms (eg, negative affect) rather than cause. Additionally, the length of follow up may not truly capture the effect or interactional quality of these risk factors.

Clinicians can use the risk factors identified in this study ${ }^{4}$ to maximise the effect of assessments and interventions. Screening should not simply assess for vulnerabilities to ED development but should also identify protective factors (eg, social supports) that might enhance specialised prevention programmes (primary to tertiary).

Mandi Newton, RN, BScN Assistant Professor School of Nursing, McMaster University Hamilton, Ontario, Canada

1 Stice E, Schupak-Neuberg E, Shaw HE, et al. Relation of media exposure to eating disorder symptomatology: an examination of mediating mechanisms. I Abnorm Psychol 1994;103:836-40.

2 Myers PN, Biocca FA. The elastic body image: the effects of television advertising and programming on body image distortions in young women. Journal of Communication 1992;42:108-33.

3 Cattarin JA, Thompson JK, Thomas C, et al. Body image, mood, and televised images of attractiveness: the role of social comparison. Journal of Social and Clinical Psychology 2000;19:220-39.

Stice E. Risk and maintenance factors for eating pathology: a meta-analytic review. Psychol Bull 2002;128:825-48.

\section{Participants}

2862 girls who were $12-21$ years of age \{mean age 15.5 y ${ }^{*}$ and did not have an ED according to DSM- $I V$ criteria.

\section{Assessment of risk factors}

Parental characteristics, mass media exposures, and other sociodemographic and lifestyle characteristics were assessed by self completed questionnaires. A validated, 36 item scale was used to assess 4 components of self esteem: social, emotional, familial, and academic.

\section{Main outcome measure}

Main outcome was ED. Girls who scored $>21$ on the Eating Attitudes Test (EAT) were referred to psychiatrists who made ED diagnoses according to DSM-IV criteria and were blinded to risk factor data. $10 \%$ of girls were unavailable for interviews; for this group, diagnoses were made on the basis of their responses to the EAT questionnaire.

\section{Main results}

90 incident cases of ED were diagnosed. Results were adjusted for age, parents' marital status, frequency of solitary eating, mass media use, socioeconomic status, body mass index, and self esteem. Independent risk factors for developing an ED were solitary eating, parental marital status other than married, and a higher level of radio use (table). When a combined score for the effect of 2 mass media variables (radio and magazine use) was added to the model, high use of both mass media (listening to radio for $>1 \mathrm{~h} / \mathrm{d}$ and reading $\geq 1$ girls' magazine per week) was associated with a higher risk of developing an ED (adjusted odds ratio 2.13, 95\% CI 1.21 to 3.76$)$.

\section{Conclusion}

Independent risk factors for developing an eating disorder were solitary eating, parental marital status other than married, and more frequent use of radio and girls' magazines.

*Information provided by author. 